\title{
Mechanism for flow generation/acceleration in dense degenerate stellar atmospheres
}

\author{
A.A. Barnaveli ${ }^{1,2}$ N.L. Shatashvili ${ }^{1,3}$
}

\begin{abstract}
The mechanism for flow generation in dense degenerate stellar atmospheres is suggested when the electron gas is degenerate and ions are assumed to be classical. It is shown, that there is a catastrophe in such system - fast flows are generated due to magnetofluid coupling near the surface. Distance over which acceleration appears is determined by the strength of gravity and degeneracy parameter. Application of this mechanism for White Dwarfs' atmospheres is examined and appropriate physical parameter range for flow generation/acceleration is found; possibility of the superAlfvénic flow generation is shown; the simultaneous possibility of flow acceleration and magnetic field amplification for specific boundary conditions is explored; in some cases initial background flow can be accelerated 100 and more times leading to transient jet formation while the Magnetic field amplification is less strong.
\end{abstract}

Keywords sun: evolution; stars: white dwarfs; plasmas

\section{Introduction}

Several recent studies were devoted to mechanisms of multi-scale equilibrium structure formations in astrophysical plasmas (Shiraishi et al 2009; Iabal et al 2008; Pino et al 2010; Mahajan \& Lingam 2015) based on

A.A. Barnaveli

${ }^{1}$ Department of Physics, Faculty of Exact \& Natural Sciences, Javakhishvili Tbilisi State University, Tbilisi 0179, Georgia

${ }^{2}$ Department of Physics, Faculty of Science, Utrecht University, 3508 TC Utrecht, The Netherlands

N.L. Shatashvili

${ }^{1}$ Department of Physics, Faculty of Exact \& Natural Sciences, Javakhishvili Tbilisi State University, Tbilisi 0179, Georgia

${ }^{3}$ Andronikashvili Institute of Physics, TSU, Tbilisi 0177, Georgia the so called Beltrami-Bernoulli (BB) class of equilibria model. Such structures were found applicable to study the heating of solar atmosphere as well as the problems of the large-scale magnetic and velocity field generation (Mahaian et al 2001; Yoshida et al 2001; Mahaian et al 2002; Mahajan et al 2005;2006);

(Ohsaki et al 2001,2002). Most investigations were limited to non-degenerate plasmas so that the constituent particles were assumed to obey the classical Maxwell-Boltzman statistics. Latest studies (Berezhiani et al 2015a; Shatashvili et al 2016) examined the possibility of the transformation of equilibrium $\mathrm{BB}$ states for the highly dense and degenerate plasmas applicable to Compact Star conditions (mean inter-particle distance is smaller than the de Broglie thermal wavelength) so that the particle energy distribution was dictated by Fermi-Dirac statistics [see (Berezhiani et al 2015b) and references therein]. Such highly dense/degenerate plasmas are also found in various astrophysical and cosmological environments as well as in the laboratories devoted to inertial confinement and high energy density physics (Dunne 2006; Yanovsky et al 2008; Mourou et al 2006; Tajima 2014).

In the theory of $\mathrm{BB}$ equilibria plasma flows play crucial role. Interestingly enough, recent observations prove that flows are widely present in solar atmosphere (Orlando et al 1995a;b; Schrijver 1999; Seaton et al 2001); (Sakai \& Furusawa 2002 ; Socas-Navarro et al 2002); (Winebarger et al 2001.2002; Aschwanden 2001); (Hansteen et al 2014; Tian et al 2014); (Cheung et al 2015; Skogsrud et al 2015) and their role in the dynamics and heating of multi-scale complexstructure solar corona is already well appreciated although the theoretical investigations were performed Mahaian et al 2001; Yoshida et al 2001; Mahajan et al 2002; Mahajan et al 2005:2006) long before the observational evidence of their existence. Flows are found crucial in 
astrophysical disks [see e.g. (Krishan \& Yoshida 2006; Zanni et al 2007; Shatashvili \& Yoshida 2011);

(Tevzadze et al 2010; Bodo et al 2015)] and their corona in inter- and extra-galactic environments. If so, then finding the steady flow generation/acceleration mechanisms in astrophysical conditions is of the utmost importance [see e.g. (Goodman 2000; Uchida 2001; Christopoulou et al 2001; Mahajan et al 2002)].

Compact astrophysical objects like white and brown dwarfs, neutron stars, magnetars with characteristic electron number densities within $\left(10^{26} \div 10^{32}\right) \mathrm{cm}^{-3}$ are the natural habitats for dense/degenerate matter Chandrasekhar 1931; Chandrasekhar 1939);

(Shapiro \& Teukolsky 1973; Begelman et al 1984);

(Michel 1982; Koester \& Chanmugam 1990);

(Michel 1991; Beloborodov \& Thompson 2007);

(Shukla \& Eliasson 2010; Shukla \& Eliasson 2011).

When a star collapses, and cools down, density of lighter elements increases affecting the total pressure/enthalpy of unit fluid element; degenerate electrons provide the dominant pressure, while the only significant source of energy is the reservoir of thermal energy in the nearly classical ideal gas of ions (see the review (Winget \& Kepler 2008) and references therein). Cooling process seems to be sensitive to outer layers/atmosphere composition, structure and their conditions. Up to now there doesn't exist a precise model of atmospheres of White Dwarfs (WDs) although recent studies show that a significant fraction of White Dwarfs are found to be magnetic with typical fields strengths $<1 K G$. At the same time, massive and cool White Dwarfs are found with much higher fields detected [see (Kepler et al 2013, and references therein)].

Magnetic WDs are considered to be stellar remnants featuring global magnetic structures with field strengths within $1 k G \div 1000 M G$ (Tremblay et al 2015a) They account for a significant part of WD population (Liebert et al 2003; Schmidt et al 2003);

(Kawka et al 2007). Most of these objects are highfield magnetic WDs, with field strengths $B>1 M G$, and a distribution of magnetic field strengths that appears to peak around $B>20 M G$ (Schmidt et al 2003; Külebi et al 2009). The origin of magnetic WDs remains elusive. At the same time, according to (Tremblay et al 2015a, and references therein), "the magnetic fields (at the $10 M G$ level) could have an influence on the structure of corresponding WD models at best only in the outermost $0.5 \%$ of the radius"; they demonstrated that convective energy transfer is seriously impeded by magnetic fields when plasma- $\beta$ becomes $<1$; the critical field strength that inhibits convection in the photosphere of WDs is in the range $B=(1-50) k G$, which is much smaller than the typical $1-1000 M G$ field strengths observed in magnetic white dwarfs, implying that these objects have radiative atmospheres. Studying the cooling process of high-field magnetic WDs $(B \gtrsim 10 M G)$, authors found that the full suppression of convection has no effect on cooling rates until the effective temperature reaches a value of around $5500 \mathrm{~K}$. In this context it is interesting that recent investigations Kawka \& Vennes 2014, and references therein) have uncovered several cool, magnetic, polluted hydrogen atmosphere WDs (DAs). Using the calcium lines they determined a surface averaged magnetic field of $B_{S}=0.335 \pm 0.003 M G$ with a velocity of $v=19.8 \pm 1.7 \mathrm{~km} / \mathrm{s}$. Using $H_{\alpha}$ they determined $B_{S}=0.331 \pm 0.004 M G$ with a velocity of $v=23.9 \pm 2.9 \mathrm{~km} / \mathrm{s}$ - the magnetic field measurements and velocities were consistent within uncertainties. It was also found that the incidence of magnetism in old, polluted WDs (DAZ) significantly exceeds what is found in the general WD population suggesting a hypothetical link between a crowded planetary system and magnetic field generation. It was shown that WD stars with such surface temperatures that convection zones develop, seems to show stronger magnetic fields than hotter stars; the mean mass of magnetic stars seems to be on average larger than the mean mass of nonmagnetic WD stars.

Pure-hydrogen-atmosphere white dwarfs convection zone study of (Tremblay et al 2015b) with the 3D simulations restricted to its upper part suggests (through hydrodynamical calculations) that the entropy of the upflows does not change significantly from the bottom of the convection zone to regions immediately below the photosphere; to calibrate 1D envelopes authors relied on this asymptotic entropy value, characteristic of the deep and adiabatically stratified layers. The calibration encompasses the convective hydrogen-line (DA) WDs in the effective temperature range $6000 \ll T_{\text {eff }}(K) \ll$ 15000 and the surface gravity range $7.0 \ll \log (g) \ll 9.0$. In typical cases for DA WDs, they found convective velocities to be of the order of $V_{z, r m s} \sim 1 \mathrm{~km} / \mathrm{s}$ at the base of the convection zone reaching maximum value of $6 \mathrm{~km} / \mathrm{s}$.

According to Hollands et al 2015) the exact fraction of WDs which have strong $(B \gtrsim 3 \mathrm{MG})$ surface magnetic fields is of order $10 \%$. Moreover, as stated above, the magnetic WDs are systematically more massive (Ferrario et al 2015). This could be partially explained by the core dynamo-generated fields; the related field strengths are right within the observed distribution of WD surface fields (Ferrario et al 2015). It is argued that many (perhaps the majority of) WDs could 
contain strong $\left(B \gtrsim 10^{6} \mathrm{G}\right)$ magnetic fields which are confined within the stellar interior and not detectable at the surface even as they cool. This is because the WD cooling timescale is shorter than its magnetic diffusion timescale $\equiv 10^{11}$ years (Cumming 2002). These fields may have very important implications for WD evolution, and for the outcome of WD mergers.

Thus, it is very important to find the mechanism for the origin and evolution of surface magnetic fields as well as studying the multi-structure dynamics of WD atmospheres in connection to both magnetic and velocity fields generation, heating/cooling; to uncover the effects of magneto-fluid couplings in outer layers of accreting stars since the dynamical evolution of their convective envelopes may define the final structure of their interior as well as of atmospheres. Knowledge of wellinvestigated similar processes in solar and sun-like star atmospheres can serve as a guidance for such investigations - the latest observations have demonstrated that the solar corona as well as the chromosphere is a highly dynamic arena replete with multiple-scale spatiotemporal structures (Aschwanden et al 2001).

Plasma flows were found to complement the abilities of the magnetic field in the creation of richness observed in Stellar atmospheres (Mahajan et al 2002; Mahajan et al 2005:2006). The most obvious process for flow generation could be the conversion of magnetic and/or thermal energy to plasma kinetic energy. Magnetically driven transient (but sudden) flow generation mechanisms permeate the literature. For a more quiescent pathway Mahajan et al 2002 applied the Bernoulli mechanism which converts thermal energy into kinetic energy, or to the general magneto-fluid rearrangement of a relatively constant kinetic energy, i.e., going from an initial high-density/low-velocity state to a low-density/high-velocity state. In above studies the double-Beltrami-Bernoulli states accessible to a two-fluid system Mahajan \& Yoshida 1998; Yoshida \& Mahajan 1999) provided the necessary framework.

As shown in (Berezhiani et al 2015a);

(Shatashvili et al 2016), when the star contracts its outer layers keep the multi-structure character although density in structures becomes defined by electron degeneracy pressure. In this respect it would be extremely important to examine the flow generation process predicted in these papers as well as the accompanying phenomena for the understanding of concrete compact objects' outer layers dynamics with degenerate plasma, specifically, for the evolution picture of magnetic White Dwarfs (WD). In present paper we present the results of detailed study of magneto-fluid coupling process in twofluid plasma with degenerate electrons exploring the acceleration mechanism, highlighting the differences with classical case and finding the appropriate physical parameter ranges for flow generation/acceleration, transient jet formation, magnetic field amplification.

\section{Model Equations for Flow generation in Dense Degenerate Stellar Atmospheres}

In (Berezhiani et al 2015a) it was developed a simplest model in which the effect of quantum degeneracy on the nature of the BB class of equilibrium states was illustrated. Emphasizing the quantum degeneracy effects, it was chosen a model hypothetical system (relevant to specific aspects of a WD) of a two-species neutral plasma with non-degenerate non relativistic ions, and degenerate relativistic electrons embedded in a magnetic field. It was assumed that, despite the relativistic mass increase, the electron fluid vorticity is negligible compared to the electron cyclotron frequency (such a situation may pertain, for example, in the pre-WD state of star evolution, or in the dynamical magnetic WD atmosphere). Study of the degenerate electron (positron) inertia effects on Beltrami States in dense neutral plasmas constituted the scope of (Shatashvili et al 2016) where the generation mechanisms of dynamical large (meso) scale were demonstrated; this scale, though present in a classical non-degenerate plasma, turns out to be degeneracy dependent and could be vastly different from its classical counterpart.

Restricting ourselves to steady state considerations our approach will be based on a straightforward application of the developed magnetofluid theory of (Mahaian \& Yoshida 1998; Mahaian et al 2001; Yoshida et al 2001; Shatashvili et al 2016) assuming that at some height of magnetic WDs surface there exist the fully ionized magnetized plasma structures such that the quasi-equilibrium two-fluid model of

(Berezhiani et al 2015a) will capture the essential physics of flow or/and magnetic field amplification. Corresponding equilibrium state equations are as follows [stating that for the non-relativistic ions and inertialess electrons there are two independent Beltrami conditions aligning ion and electron generalized vorticities along their respective velocities]:

$\mathbf{b}=a N\left[\mathbf{V}-\frac{1}{N} \nabla \times \mathbf{b}\right]$

$\mathbf{b}+\nabla \times \mathbf{V}=d N \mathbf{V}$,

where $\mathbf{b}=e \mathbf{B} / m_{i} c$ and it was assumed, that electron and proton laboratory-frame densities are nearly equal $-N_{e} \simeq N_{i}=N \quad$ [rest-frame density $n_{e, i}=$ 
$N_{e, i} / \gamma\left(\underline{\mathrm{V}}_{e, i}\right)$ with $\gamma\left(\underline{\mathrm{V}}_{e, i}\right)$ being a Lorentz factor for electrons/ions]; here $a$ and $d$ are dimensionless constants related to the two invariants: the magnetic helicity $h_{1}=\int(\mathbf{A} \cdot \mathbf{b}) d^{3} x$ and the generalized helicity $h_{2}=\int(\mathbf{A}+\mathbf{V}) \cdot(\mathbf{b}+\nabla \times \mathbf{V}) d^{3} x$ of the system with A being the dimensionless vector potential. Equations (11) and (2) are written in terms of normalized one fluid variables: the fluid velocity $\mathbf{V}$ and the current $\mathbf{J}=\nabla \times \mathbf{b}$ (via Ampere's law) when the electron and the ion speeds are given by $\mathbf{V}_{e}=\mathbf{V}-(1 / N) \nabla \times \mathbf{b}$, and $\mathbf{V}_{i}=\mathbf{V}$, respectively (the electrons, as stated above, are assumed to be inertia less). Notice, that in this approximation the electron vorticity is primarily magnetic (b) while the ion vorticity has both kinematic and magnetic parts $(\mathbf{b}+\nabla \times \mathbf{V})$. Also, in above equations, the density is normalized to $N_{0}$ (the corresponding restframe density is $n_{0}$ ); the magnetic field is normalized to some ambient $B_{0}$; all velocities are measured in terms of the corresponding Alfvén speed $V_{A}=B_{0} / \sqrt{4 \pi N_{0} m_{i}}$ ; all lengths [times] are normalized to the skin depth $\lambda_{i}\left[\lambda_{i} / V_{A}\right]$, where $\lambda_{i}=c / \omega_{p i}=c \sqrt{m_{i} / 4 \pi N_{0} e^{2}}$. We would like to comment here that the fundamental result of vortex dynamics of any ideal fluid is that it implies a topological invariant, the helicity (generaliced helicity), i.e. such an invariance forbids the creation of vorticity (generalized vorticity) from no-vorticity (no-generalized vorticity) state of the two-fluid system (Mahajan 2003; Mahaian \& Yoshida 2010; Pino et al 2010),

(Bhattacharjee et al 2015), and of more general multispecies ideal plasma (Mahajan \& Lingam 2015). The reason of such invariance lie deep in the Hamiltonian structure governing the dynamics of the ideal fluid Mahaian \& Yoshida 1998; Morrison 1998),

(Arnold \& Khesin 1998) and is independent of compressibility; however this constraint gets broken for non-ideal multi-species plasmas. In latter case all the corresponding helicities become dynamical, hence, defining the fate of the system locally

\section{(Mahajan et al 2005:2006).}

To define an equilibrium state (the stationary solution of the dynamical system) the Beltrami conditions (11) and (2) shall be supplemented by the Bernoulli constraint relating the density with the flow kinetic energy through degeneracy pressure, which reads as

$\nabla\left(\beta_{0} \ln N+\mu_{0} \sqrt{1+P_{F}^{2}} \gamma+\frac{V^{2}}{2}\right)=0$

where $\beta_{0}$ is the ratio of the thermal pressure to the magnetic pressure, $\mu_{0}=m_{e} c^{2} / m_{i} V_{A}^{2}$ and for the electron fluid Lorentz factor $\gamma_{e} \simeq \gamma(\mathbf{V})$ was used. Since the normalized Fermi momentum of electrons $P_{F}=p_{F} / m_{e} c=\left(N N_{0} / n_{c} \gamma\right)^{1 / 3}$ is a function of the density $N$ (here $n_{c}=5.9 \times 10^{29} \mathrm{~cm}^{-3}$ is the critical number-density at which the Fermi momentum equals $\left.m_{e} c\right)$, the system of equations (11)-(2)-(3), together with the automatically satisfied equilibrium continuity equation $[\nabla \cdot(N \mathbf{V})=0]$ and the divergence free condition for magnetic field $[\nabla \cdot \mathbf{b}=0]$, form a fully specified equilibrium - a complete system to determine $\mathbf{N}$, $\mathbf{V}$ and $\mathbf{b}$. Notice, that for significant $P_{F}(N)$ [relevant to WD's conditions] in (3) the degeneracy pressure can be much bigger than the thermal pressure (measured by $\beta_{0}$ ) and, hence, the cold (low $\beta_{0}$ ) degenerate electron gas can sustain a qualitatively new state: there exists a nontrivial Double BB equilibrium at zero/low temperatures (Berezhiani et al 2015a) [in contrast to the classical zero-beta plasmas, where only the relatively trivial, single Beltrami states are accessible (Yoshida et al 2001)]. We will show below that, when gravity effects are also taken into account, exactly this interesting departure from classical situation makes it possible to couple the magnetic and velocity fields in degenerate dense and cold WD stars outerlayers / atmospheres discussed in the introduction.

Bernoulli condition (3) introduces a qualitatively new player in the equilibrium balance - the spatial variation in electron degeneracy energy (proportional to $\mu_{0}=m_{e} c^{2} / m_{i} V_{A}^{2}$ ) could increase / decrease the fluid kinetic energy (measured by $V^{2}$ ) or plasma $\beta_{0}$ in the corresponding region of star's outer layer / atmosphere converting Fermi energy to the kinetic/thermal energy or vice versa; it could also forge a re-adjustment of kinetic energy from a high-density/low-velocity plasma to a low-density/high-velocity plasma. Related energy transformations, mediated through classical gravity in solar atmosphere, were discussed in Mahajan et al $(2002,2006)$. While for the special class of magnetic WDs study of (Berezhiani et al 2015a) predicted that the electron degeneracy effects can be both strong and lead to the anti-correlation between density and flow speeds - the generated flow gets locally super-Alfvénic in contradistinction to non-degenerate, thermal pressure dominated Solar Atmosphere plasma (with local plasma $\beta<1$ ) for which the maximal velocity due to the magneto-Bernoulli mechanism was found to be only locally sub-Alfvénic.

In present paper we go further and construct the detailed solutions of (1] 3) with gravity taken into account for concrete parameters relevant to magnetic WDs to show the explicit effects of degeneracy on two-fluid BB structures when star contracts and cools down. First we rewrite these equations with inclusion of classical (Newtonian) gravity for nonrelatvistic flows $[\gamma(\mathbf{V}) \sim 1$ 
justified by observations] as follows:

$\mathbf{b}=a N\left[\mathbf{V}-\frac{\kappa}{N} \nabla \times \mathbf{b}\right]$,

$\mathbf{b}+\kappa \nabla \times \mathbf{V}=d N \mathbf{V}$

$\nabla\left(\beta_{0} \ln N+\mu_{0} \sqrt{1+P_{F}^{2}}-\frac{R_{A}}{R}+\frac{V^{2}}{2}\right)=0$

where $R$ is a radial distance from the center of WD normalized to its radius $R_{W}\left[\sim(0.008-0.02) R_{\odot}\right]$ and $R_{A}=G M_{W} / R_{W} V_{A}^{2}$ (here $G$ is the gravitational constant, $M_{W}$ is a WD mass); dimensionless parameter $\kappa=\lambda_{i} / R_{w}$.

For typical cold magnetic WDs with the degenerate electron densities $\sim\left(10^{25}-10^{29}\right) \mathrm{cm}^{-3}$; magnetic fields $\sim\left(10^{5}-10^{9}\right) G$, and temperatures $\sim(40000-$ 6000) $K$ (and, hence, with corresponding Alfvén speed $\left.V_{A} \sim\left(10^{4}-10^{6}\right) \mathrm{cm} / \mathrm{s}\right)$ yielding $\beta_{0} \sim\left(10^{6}-10^{0}\right)$, $R_{A} \sim\left(10^{8}-10^{4}\right)$ and $\mu_{0} \sim\left(10^{10}-10^{6}\right) \gg \beta_{0}$, $\lambda_{i} \sim\left(10^{-5}-10^{-7}\right) \mathrm{cm}$ (being rather small). Comparing the terms in (6) for above parameters, one can see the dominance of electron fluid degeneracy pressure in equilibrium balance. Then, neglecting the first term related to ion fluid pressure, Eqs. (44), (5), (6) can be easily manipulated to obtain:

$\frac{\kappa^{2}}{N} \nabla \times \nabla \times \mathbf{V}+\kappa \nabla \times\left[\left(\frac{1}{a N}-d\right) N \mathbf{V}\right]+\left[1-\frac{d}{a}\right] \mathbf{V}=0$

$N=\left[\frac{\left(\left[\frac{R_{A}}{R}-\frac{R_{A}}{R_{0}}\right]-\left[\frac{\mathbf{V}^{2}}{2}-\frac{\mathbf{V}_{\mathbf{0}}{ }^{2}}{2}\right]+\alpha \mu_{0}^{2}\right)^{2}}{a_{0}^{2} \mu_{0}^{2}}-\frac{1}{a_{0}^{2}}\right]^{3 / 2}$,

with $a_{0}=\left(n_{0} / n_{c}\right)^{1 / 3}$ and $\alpha=\sqrt{1+a_{0}^{2} N_{0}^{2 / 3}}$; here subscript " 0 " is used for the height from stellar surface where the boundary conditions are applied. These equations provide us with a closed system of four equations in four variables $(N, \mathbf{V})$. Once this is solved with appropriate boundary conditions for magnetic WDs, one can invoke (5) to calculate $\mathbf{b}$. The reader can find the solution for the similar mathematical problem relevant to the non-degenerate (solar atmosphere) case in (Mahajan et al 2002).

We must emphasize, that these time-independent equations are not suitable for studying the heating/cooling processes, the latter requires a fully timedependent treatment; main thrust of this communication is to uncover the mechanisms that create/accelerate flows and amplify the magnetic fields in outer layers of WDs. Since $P_{F}=a_{0} N^{1 / 3}$ is a function of Fermi energy (and, hence, a function of density), Bernoulli equation (6) relates density with flow kinetic energy and classical gravity [Eq. (8)] in a qualitatively different way from classical case. Also, since for most class of magnetic WDs outer-layer/atmosphere conditions the degeneracy pressure is larger than the thermal pressure in Eq.(6), the new results are eventually expected in this very limit of star evolution while its contruction/accretion. At the same time it is evident that due to the same reason the influence of discussed nonlinear coupling process on the cooling rate of compact object is automatically eliminated; possibly this proves the findings of (Tremblay et al 2015a) about the non-affected cooling process relevant for most of cold magnetic WDs.

\section{Simulation Results for Flow generation in Dense Degenerate Stellar Atmospheres}

To explore the mechanisms for flow and magnetic field generation / amplification in dense degenerate stellar atmospheres we have carried out a one-dimensional extensive simulation experiments (the relevant dimension being the height "Z" from the center of WD; $Z_{0}=R_{W}+\Delta R$ is the surface at which the boundary conditions are applied) of the coupled nonlinear system of equations (7- 8) for a variety of boundary conditions. The results are presented in Figures 1-8. These are the plots of different physical characteristic quantities as functions of height. For all our runs the boundary conditions $\left|\mathbf{b}_{0}\right|=1$ and $V_{0}=a^{-1} V_{A, 0}$ (with $a \sim d ; V_{x, 0}=V_{y, 0}=V_{z, 0}$ in Cartesian Geometry) were imposed (see equations (4) and (5D) and due to the limitations of the code the values chosen for $\kappa$ (measure of the strength of two-fluid Hall currents) were larger than their actual values for WD atmospheres but, as seen from the illustrative results, the physical tendency of the nonlinear couplings are well seen even for such assumptions. We have chosen two different DB parameters $(d \sim a)$ limits to start with various initial set-ups; e.g. (i) choice of $d \sim a \gg 1$ means that our initial flow is locally subAlfvénic (strongly magnetic WD outer layer) while (ii) the opposite choice of $d \sim a \ll 1$ would mean that we are dealing with the super-Alfvénic flow to start with (weakly magnetic WD outer layer). Notice, that since densities of WDs' outer layer / atmosphere degenerate plasma are very high the Alfvén speeds can be rather low locally and we can deal with both extreme cases for initial setups - even the weak initial 
flow can become super-Alfvénic locally. It is important to emphasize here that even the ion skin depth is very small, its contribution may become very crucial [see for solar case (Mahajan et al 2001; Yoshida et al 2001)]; this short scale associated with related singular perturbation [first term of Eq. (77)] of the system introduces the new channel for energy transformations Mahaian et al 2002; Mahaian et al 2005;2006; Berezhiani et al 2015a; Shatashvili et al 2016). If one ignores this short scale (neglecting both Hall term and two-fluid effects) one would end up creating the significant radial flows at much larger distances from the stellar surface or the process of flow acceleration will be completely eliminated from the dynamics [see, for instance, the 2.5D-simulation results for magneto-fluid couplings for classical solar case - Fig.12 of Mahajan et al 2006].

To better illustrate the coupling process we have picked up several sets of our runs. The simulation results in Figures 1 and 2 for various physical quantities versus height consist of 3 frames (e.g Figs 1a and 1b; Figs 1c and 1d; Figs 1e and 1f), each consisting of two pictures: one for the density and magnetic field and the other for the velocity field. In each frame there are 3 sets of curves corresponding, respectively, to $\kappa=10^{-2}, \kappa=10^{-4}, \kappa=10^{-6}$. Parameters defining different frames are (we are giving them in the order $\left.a=d, n_{0}, B_{0}, V_{A}\right)$ as follows: for Fig.1: (1) $10^{-1}, 10^{26} \mathrm{~cm}^{-3}, 10^{8} \mathrm{G}, 15 \mathrm{~km} / \mathrm{s}$ implying $a_{0}=(1 / 6)^{1 / 3} 10^{-1}, V_{0}=10 V_{A}=150 \mathrm{~km} / \mathrm{s}$, $\mu_{0}=10^{7} \quad$ (Figs.1a and 1b); (2) $10^{-1}, 10^{26} \mathrm{~cm}^{-3}, 3$. $10^{7} \mathrm{G}, 5 \mathrm{~km} / \mathrm{s}$ implying $a_{0}=(1 / 6)^{1 / 3} 10^{-1}, V_{0}=$ $10 V_{A}=50 \mathrm{~km} / \mathrm{s}, \mu_{0}=10^{8} \quad$ (Figs.1c and 1d);

(3) $10^{-1}, 10^{26} \mathrm{~cm}^{-3}, 10^{7} \mathrm{G}, 1.5 \mathrm{~km} / \mathrm{s}$ implying $a_{0}=$ $(1 / 6)^{1 / 3} 10^{-1}, \quad V_{0}=10 V_{A}=15 \mathrm{~km} / \mathrm{s}, \quad \mu_{0}=10^{9}$ (Figs.1e and 1f) and for Fig.2: (4) $10,10^{27} \mathrm{~cm}^{-3}, 3$. $10^{8} \mathrm{G}, 15 \mathrm{~km} / \mathrm{s}$ implying $a_{0}=(1 / 6)^{1 / 3} 10^{-2 / 3}, V_{0}=$ $0.1 V_{A}=1.5 \mathrm{~km} / \mathrm{s}, \mu_{0}=10^{7}$ (Figs.2a and $2 \mathrm{~b}$ );

(5) $10,10^{27} \mathrm{~cm}^{-3}, 10^{8} \mathrm{G}, 5 \mathrm{~km} / \mathrm{s}$ implying $a_{0}=(1 / 6)^{1 / 3} 10^{-2 / 3}, V_{0}=0.1 V_{A}=0.5 \mathrm{~km} / \mathrm{s}, \mu_{0}=$ $10^{8}$ (Figs.2c and 2d); (6) $10,10^{27} \mathrm{~cm}^{-3}, 3 \cdot 10^{7} \mathrm{G}$, $1.5 \mathrm{~km} / \mathrm{s}$ implying $a_{0}=(1 / 6)^{1 / 3} 10^{-2 / 3}, \quad V_{0}=$ $0.1 V_{A}=0.15 \mathrm{~km} / \mathrm{s}, \mu_{0}=10^{9}$ (Figs.2e and $2 \mathrm{f}$ ).

In Fig.3 we give the simulation results for $n_{0}=$ $10^{25} \mathrm{~cm}^{-3}\left[a_{0}=(1 / 6)^{1 / 3} 10^{-4 / 3}\right]$ for $B_{0}=3 \cdot 10^{6} \mathrm{G}, a=$ $d=10$ (implying $V_{A}=1.5 \mathrm{~km} / \mathrm{s}, \mu_{0}=10^{9}$ ) illustrating that for such surface conditions starting from $0.15 \mathrm{~km} / \mathrm{s}$ speed flow we arrive to $60 \mathrm{~km} / \mathrm{s}$ accelerated (400 times!) flow but at lower densities of $\sim 10^{24} \mathrm{~cm}^{-3}$. We can clearly see from this picture that in low-density degenerate case the flow-generation picture practically repeats the classical case results (? ); at the same time one can distinguish the important difference - there is
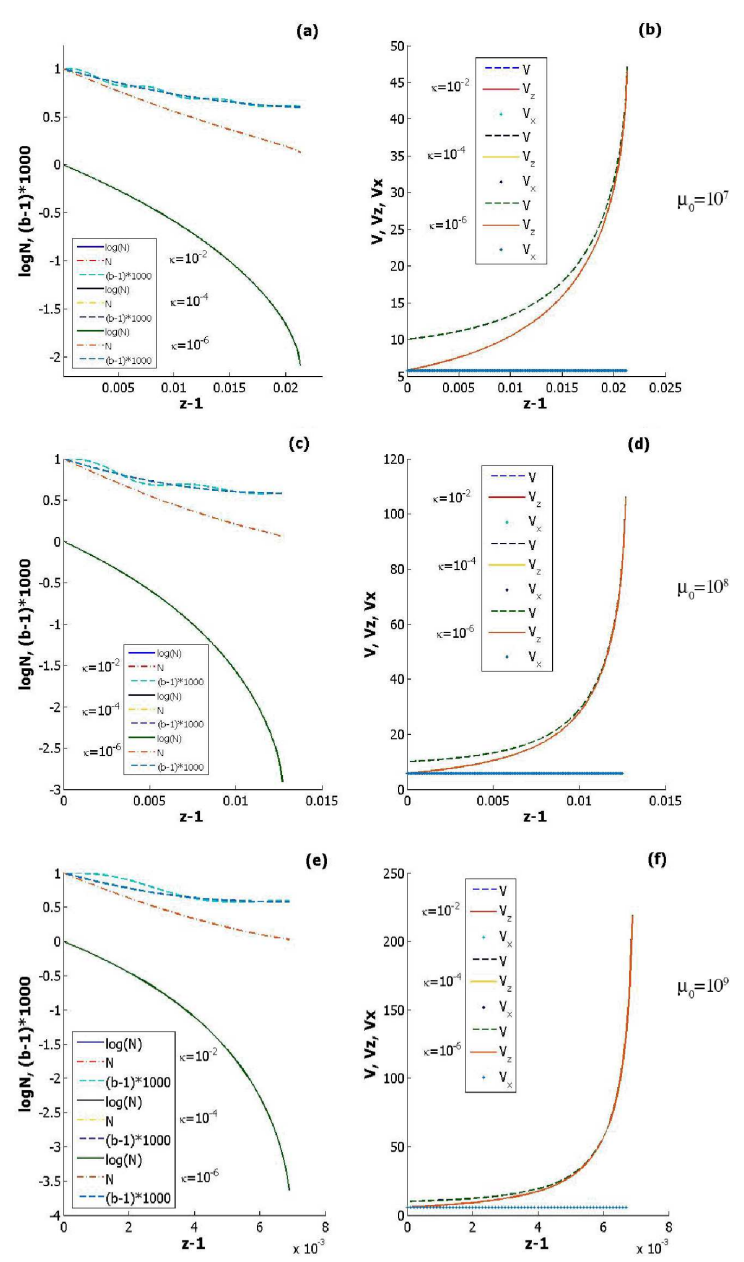

Fig. 1 Plots for density, magnetic fields and velocity vs height for $n_{0}=10^{26} \mathrm{~cm}^{-3}\left[a_{0}=(1 / 6)^{1 / 3} 10^{-1}\right]$ and DB parameter $a=d=0.1$ at various values of $\mu_{0}$ : (a) and (b) are for $\mu_{0}=10^{7}$ and $R_{A} / \mu_{0}=0.05$; (c) and (d) are for $\mu_{0}=$ $10^{8}$ and $R_{A} / \mu_{0}=0.1$ and (e) and (f) are for $\mu_{0}=10^{9}$ and $R_{A} / \mu_{0}=0.2$. The 3 sets of curves represent results for $\kappa=$ $10^{-2}, \kappa=10^{-4}, \kappa=10^{-6}$, respectively. $V_{y}$ is not displayed due to its similar behavior to $V_{x}$. For all $\kappa$-s one clearly sees the dominance of radial velocity - initial flow accelerates significantly. Magnetic field components show oscillating character although its energy doesn't change much at these heights for chosen Beltrami parameters. 

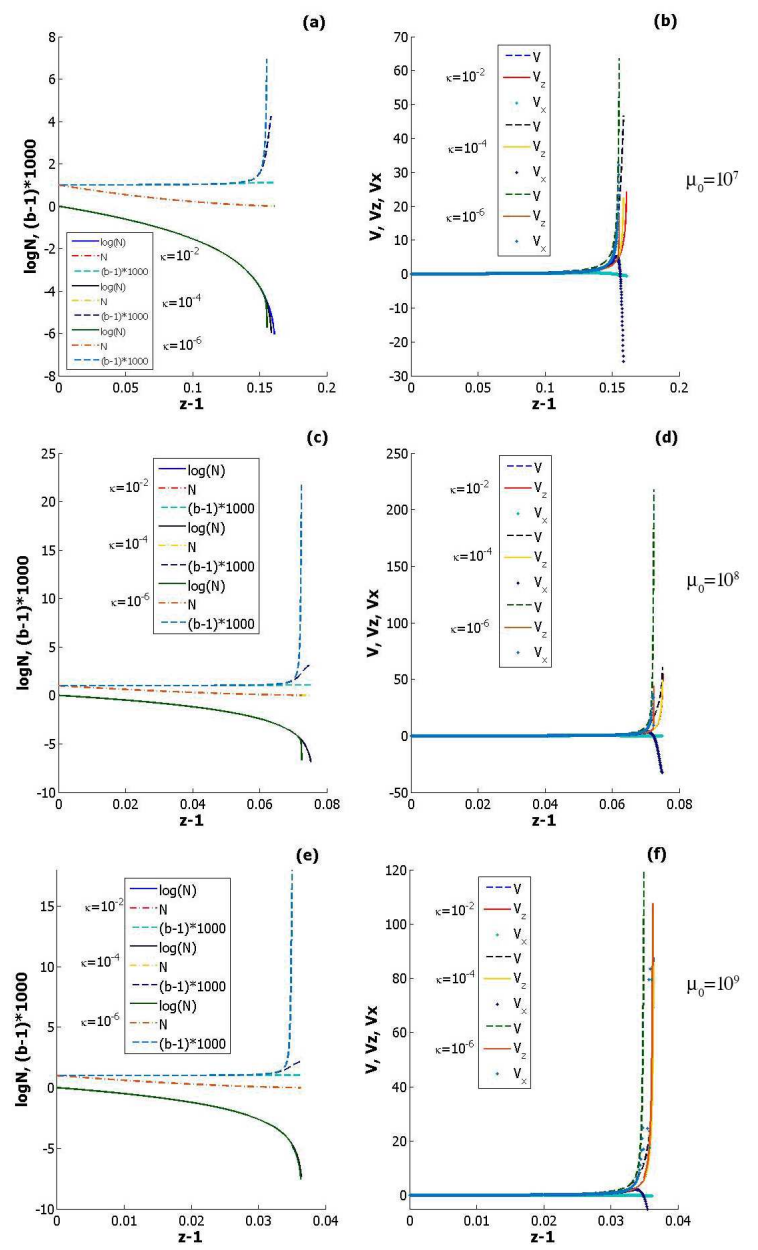

Fig. 2 Plots for density, magnetic fields and velocity vs height for $n_{0}=10^{27} \mathrm{~cm}^{-3}\left[a_{0}=(1 / 6)^{1 / 3} 10^{-2 / 3}\right]$ and DB parameter $a=d=10$ various values of $\mu_{0}$ : (a) and (b) are for $\mu_{0}=10^{7}$ and $R_{A} / \mu_{0}=0.05$; (c) and (d) are for $\mu_{0}=10^{8}$ and $R_{A} / \mu_{0}=0.1$ and (e) and (f) are for $\mu_{0}=10^{9}$ and $R_{A} / \mu_{0}=0.2$. The 3 sets of curves represent results for $\kappa=10^{-2}, \kappa=10^{-4}, \kappa=10^{-6}$, respectively. $V_{y}$ is not displayed due to its similar behavior to $V_{x}$. For all $\kappa$-s initial flow accelerates significantly. There is a clear evidence of magnetic field amplification together with flow acceleration. a slight enhancement of the magnetic field as well! Although this enhancement $\left(\sim 5 \cdot 10^{3} G\right)$ is small compared to background field its catastrophic character of the increment introduces the short-scale in the magnetic field structure which could trigger the severe transformations in the initial equilibrium system (see e.g. (? )). Also, later on, dynamically, such flow-magnetic field region may become a boundary condition for a separate coupling process leading to more complicated picture of flow generation at the end.

The simulations show that, like in the solar case, for small $\kappa$-s there exists some height at which the density begins to drop dramatically (sometimes 400500 times) with a corresponding sharp rise in the flow speed and, often [for $a \sim d<1$ ] in magnetic field as well. Effect is stronger for high $\mu_{0}$ (initially high density and low magnetic field region) degenerate plasmas. It is also obvious, that since the DB structure scales are small compared to $R_{W}$ in outer layers / atmosphere of the WD (where our model applies) the gravity contribution, like in the solar case Mahajan et al 2002; Mahajan et al 2005:2006), determines the radial distance in WDs outer layer over which the fast and catastrophic acceleration of flow may appear due to the magneto-fluid coupling. At the heights where the flows are insignificant (at very short distances from the WDs surface) gravity controls the stratification but as we approach the flow blow-up distances (the flow becomes relatively strong) the self-consistent magneto-Bernoulli processes (with degeneracy effects included) take over and control the density (and hence the velocity or/and magnetic field) stratification.

We have also verified the analytical prediction of (Berezhiani et al 2015a) for maximal flow speed
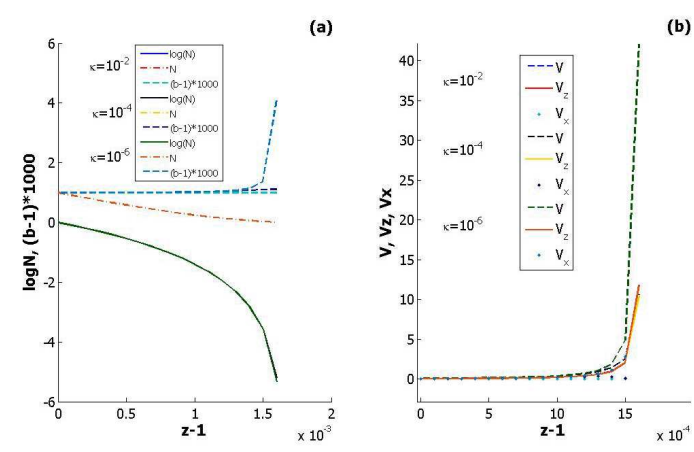

Fig. 3 Plots for density, magnetic fields and velocity vs height for $n_{0}=10^{25} \mathrm{~cm}^{-3}\left[a_{0}=(1 / 6)^{1 / 3} 10^{-4 / 3}\right]$ and DB parameter $a=d=10$ at $\mu_{0}=10^{9}$. Initial flow with $0.15 \mathrm{~km} / \mathrm{s}$ speed accelerates 450 times reaching the speed of $60 \mathrm{~km} / \mathrm{s}$; process is accompanied by the magnetic field amplification. 
[achieved after acceleration] to be super-Alfvénic . Interestingly enough, as numerical simulation analysis showed, even for unrealistic Hall term strength parameter $\kappa \sim 10^{-6}$ at specific boundary conditions we can see the tendency of accelerated flow to become at the end locally almost super-Alfvénic (starting from sub-Alfvénic); for instance, initially subAlfvénic flow $\left(V_{A}=5 \mathrm{~km} / \mathrm{s} ; a=d=10\right)$ with speed $V_{0}=0.5 \mathrm{~km} / \mathrm{s}$ and density $\sim 10^{27} \mathrm{~cm}^{-3}$ accelerates sharply (2500 times) up to $1250 \mathrm{~km} / \mathrm{s}$ becoming slightly super-Alfvénic locally at $Z-Z_{0}=0.07 R_{W}$ and with lower density $\leq 10^{23} \mathrm{~cm}^{-3}$ (Figs. $2 \mathrm{~b}$ and 2c); it is clear that accelerated flow remains strongly super-Alfvénic locally when starting from being super-Alfvénic initially due to severe drop in density - see Fig.1). Thus, on can expect that lowering $\kappa$ the chances of ending up with super-Alfveénic accelerated flows increase (see Fig.7 and Fig.8); the higher is the initial density/the lower the initial magnetic field better could be the picture ( $\mu_{0}$ goes up with density/inverce magnetic field). In some cases the magnetic energy remained unchanged (although the magnetic fields components would change self-consistently, assuring the varying magnetization properties), while in some cases (see e.g. Fig.2 and Fig.3) the magnetic field energy was changed sharply; in few cases its change was wave-like [like e.g. in Fig.1]. For DB parameter $a=10(>1)$ one can distinguish the enhancement of $\mathbf{B}$-field for all densities $\geq 10^{25} \mathrm{~cm}^{-3}$, this is well seen in Fig.3. It is evident, that the sharp decrease in density with a corresponding sharp rise in the flow speed (and also in magnetic field) is the expression of Bernoulli constraint imposed by the magneto-fluid equilibrium condition. Notice, that we have neglected the thermal pressure - keeping it would not change the main results due to the observationally evident very high degeneracy parameter $\mu_{0}$ in compact stellar outer layers, thus, controlling the equilibrium state.

In Figures 4 and 5 we present the results for blow up distance (left frame) and blow-up velocity (right frame) versus DB parameter $a \sim d$ for different boundary conditions relevant to Figs 1 and 2. We can clearly see that the final velocity decreases with DB parameter and depends strongly on the degeneracy parameter/magnetism of the stellar outer layer. The smaller is the degeneracy parameter $\mu_{0}$ (low density/high magnetism) lower is the final speed, blow-up distance is farther and vice-versa - higher the degeneracy parameter $\mu_{0}$ (high density/low magnetism) higher is the final speed for the same $a$, blow-up distance is closer to stellar surface. Also, from both figures one can see, that for the same DB parameter $a \ll 1$ both Blow-up Distances and Blow-up Velocities significantly differ for different degeneracy parameter/magnetism (lower the DB parameter more different they are) while for greater DB parameter such difference disappears - their values become similar for all $\mu_{0}$ already for $a=100$. Hence, for large DB parameters both Blow-up Distance and Blowup Velocity are practically independent of $a \sim d$. Such effect is stronger for larger initial densities (see Fig.5).

We have performed the detailed analysis to get more clear information about the magneto-fluid coupling efficiency - to observe the dependence of flow/magnetic field blow-up process on the Hall Current strength parameter $\kappa$. For larger $\kappa$-s flows mostly tend to be radial, while for smaller $\kappa$-s Double-Beltrami effects enter the play and the transverse components of these vector-fields become more important. Situation could change when we deal with the time-dependent dynamical model with dissipation (e.g. when taking into account the cooling/heating of the plasma); these processes would change degeneracy parameter $\mu_{0}$ as well as the plasma $\beta_{0}$ locally shifting the velocity/magnetic field blow-up distance at lower/upper heights or eliminating it altogether - one can observe in Figs 1 and 2 that as $\mu_{0}$ goes up (density [magnetic field] increases [decreases]) the fall in density [with accompanying amplification of velocity/magnetic field] becomes smoother. At fixed $\mu_{0}$ the blow-up distances and speeds are not changed much with $\kappa$ for lower DB parameters $(a \sim d<1)$ (Fig.6), thus, indicating, that the efficiency of this process is less sensitive to smallness rate of Hall Current strength $\kappa$ (important is that it is not zero). At higher $a \sim d[>1]$ for larger $\mu_{0}$ one observes the increase of amplification phenomenon at smaller $\kappa$-s - see Figs $7 \& 8$ ); effect is stronger for higher initial densities.

Notice that final velocities get larger with $V_{0}[\mathrm{~km} / \mathrm{s}]$ $\sim a^{-1} V_{A}$. For instance, initial flow with speed $1.5 \mathrm{~km} / \mathrm{s}$ ends up acquiring a high speed $\sim 105 \mathrm{~km} / \mathrm{s}$ (Figs. $2 \mathrm{a}$ and $2 \mathrm{~b}$ ) at height $\sim 0.15 R_{W}$ while initial flow with speed $150 \mathrm{~km} / \mathrm{s}$ ends up acquiring a high speed
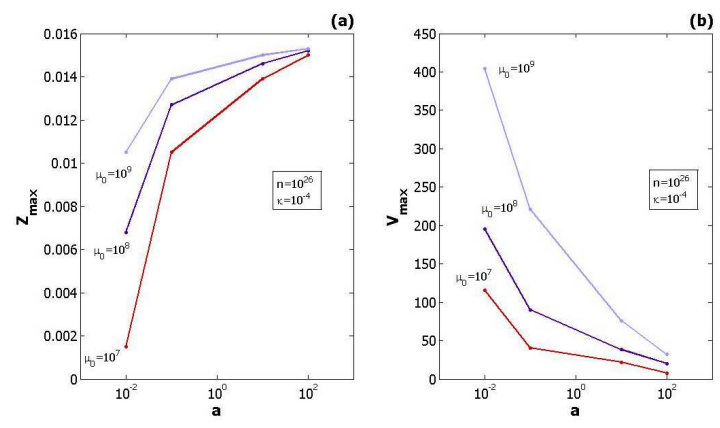

Fig. 4 (a) Blow-up distance and (b) blow-up velocity versus DB parameter $a \sim d$ for initial density $n_{0}=10^{26} \mathrm{~cm}^{-3}$. The smaller the $\mu_{0}$ lower is the final speed, as well as smaller is the blow-up distance. For large $a$ both blow-up distances and velocities come closer to each other for different $\mu_{0}-\mathrm{s}$. 

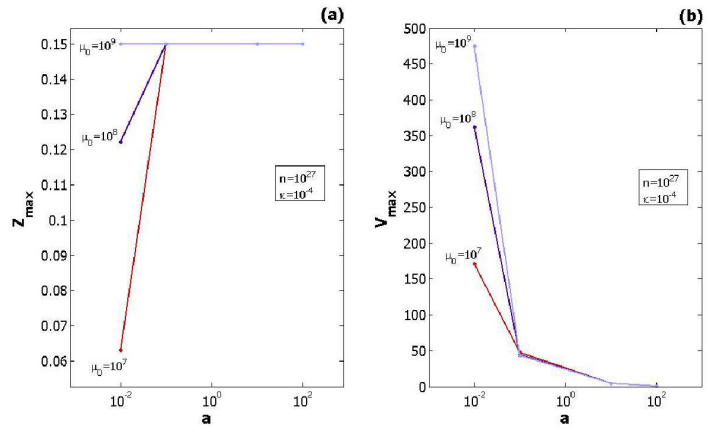

Fig. 5 Blow-up (a) distance and (b) velocity versus DB parameter $a \sim d$ for initial density $n_{0}=10^{27} \mathrm{~cm}^{-3}$. For large $a>10^{-1}$ both blow-up distances and velocities become practically independent of $a$ being similar for all $\mu_{0}-\mathrm{s}$.


Fig. 6 Blow-up (a) distance and (b) velocity vs Hall-term strength $\kappa$ for $n_{0}=10^{26} \mathrm{~cm}^{-3}$ and $a=d=0.1$. The smaller the $\mu_{0}$ lower is the final speed and smaller is the blow-up distance. For fixed $\mu_{0}$ blow-up process is less sensitive to changes in $\kappa$.
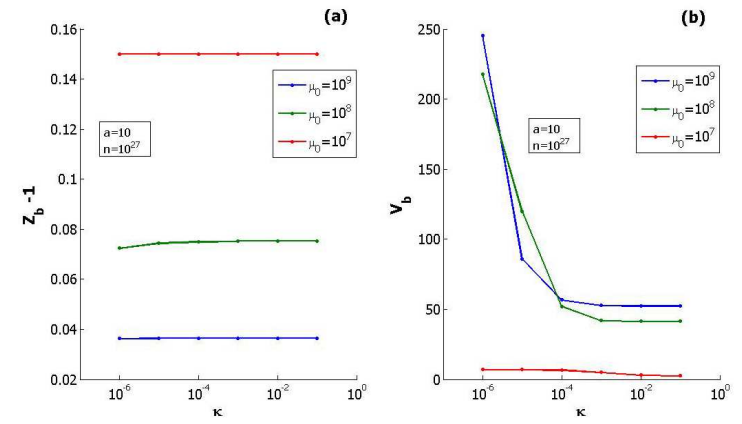

Fig. 7 Blow-up (a) distance and (b) velocity vs $\kappa$ for $n_{0}=10^{27} \mathrm{~cm}^{-3}$ and $a=d=10$. For fixed $\mu_{0}$ blow-up process is more sensitive to changes in $\kappa$ at higher $\mu_{0}$ (lower initial magnetic field). There is a clear tendency for initial flow to become Super-Alfvénic at blow-up. $\sim 750 \mathrm{~km} / \mathrm{s}$ (Figs. 1a and 1b) at height $\sim 0.02 R_{W}$ but at much lower densities compared to their initial one.

At this end we would like to summarize that there is an intrinsic tendency of velocity / magnetic field amplification in our two-fluid system of classical ions and degenerate elecrons due to magneto-fluid coupling. Also it is important to emphasize that such coupling effect leads the system to the blow-up - the catastrophic amplification of magnetic/velocity vector-fields - which can not be ignored in the dynamics of WD's outer layers with degenerate electrons. Figs 4-8 clearly show, that the dependence of both vector-fields (velocity and magnetic) during the blow-up process on DB $[a, d]$ and degeneracy $\left[\mu_{0}\right]$ parameters is fundamentally crucial: (1) for the different degeneracy parameters the final results are different for the same $a \sim d$ (unless the latter is much greater than one) while (2) for the same degeneracy parameters final results may change considerably for different $a$-s, thus, indicating that the blow-up process is very sensitive, in fact, to both the degeneracy state of the system and the magneto-fluid coupling. At the same time, since for greater DB parameters the final blow-up speeds (blow-up distances) come closer to each other for different $\mu_{0}$-s it becomes practically impossible to distinguish the realistic scenario (select the corresponding boundary conditions) of velocity/magnetic field amplification when comparing to observations. If one ignores the flow term in Bernoulli constraint (6) one ends up finding the radial flows with very low magnitude, and, like in Solar case (Mahajan et al 2002), there will be no region of sharp rise; also generated flows/magnetic fields will achieve distinguishable energies on much longer distances from the surface than the correct Bernoulli constraint would maintain it. Thus, to find correctly the final values of amplified vector-fields and also to determine the correct heights where such catastrophic transformations would

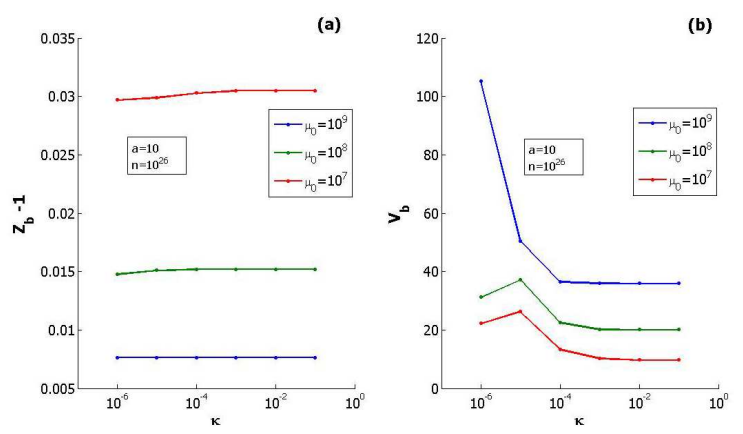

Fig. 8 Blow-up (a) distance and (b) velocity vs $\kappa$ for $n_{0}=10^{26} \mathrm{~cm}^{-3}$ and $a \sim d=10$. 
take place one has to perform the magneto-fluid coupling analysis.

\section{Summary and Conclusions}

In present manuscript we extended the studies of recent papers (Berezhiani et al 2015a; Shatashvili et al 2016) and, based on the systematic simulation experiments, showed that the degeneracy effects are significant for specific class of dense stellar atmospheres/outer layers dynamics, specifically, for the structure formation phenomena there - we suggest that when studying the evolution of the compact objects, flow effects cannot be ignored since their catastrophic generation close to the surface may determine the further evolution of stars and their atmospheres. In addition, as observations indicate, for evolution picture, specifically in case of magnetic WDs, it is of utmost importance to know the magnetic fields dynamics/fate. In this respect our study indicates that for specific WD-surface conditions it is possible to amplify the magnetic fields in combination with flow generation/acceleration. This simultaneous possibility of amplifying catastrophically flow and magnetic fields is a demonstration of magneto-fluid coupling - here the degeneracy has a striking effect. The distance over which amplification appears is determined by the strength of gravity and degeneracy parameter; Double-Beltrami parameters also play the crucial role in the efficiency of coupling. Examining the concrete applications for cool WDs atmospheres we found the appropriate physical parameter ranges for the flow generation/acceleration and the magnetic field amplification; process is less sensitive to surface temperature since the major pressure is due to degeneracy. The possibility of locally Super-Alfvénic flow generation is found for various surface parameters of WDs this is a remarkable result compared to Sun for which the generated flows (by the same mechanism) were previously found to be locally sub-Alfvénic for low-beta plasmas (Mahajan et al 2002). For specific conditions of WD's outer layers initial sub-Alfvénic flow can be accelerated 100 and more times leading to the transient jet formation. The results of given study can be applied for the understanding of the origin and evolution of White Dwarfs, dense compact objects; for their cooling and accretion dynamics for which, as demonstrated in present study, the magneto-fluid coupling processes when degeneracy effects are taken into account, specifically when time-dependency is also included, become determining and crucial.

\section{Acknowledgements}

NLS acknowledges special debt to the Abdus Salam International Centre for Theoretical Physics, Trieste, Italy. AAB thanks Doctors A. Tevzadze and G. Mamatsashvili for the valuable discussions.

\section{References}

Arnold, V.I. and Khesin, B.A. Topological Methods in Hydrodynamics (Springer, New York, 1998).

Aschwanden, M. J., Poland, A. I., \& Rabin, D. M. ARA\&A, 39, 175 (2001).

Aschwanden, M. J. Astrophys. J., 560, 1035 (2001).

Begelman, M.C., Blandford, R.D., and Rees, M.D. Rev. Mod. Phys. 56255 (1984).

Beloborodov, A.M. and Thompson, C. Astrophys. J.657, 967 (2007).

Berezhiani, V.I., Shatashvili, N.L. and Mahajan, S.M. Phys. Plasmas 22, 022902 (2015a).

Berezhiani, V.I., Shatashvili, N.L. and Tsintsadze, N.l. Physica Scripta 90(6), 068005 (2015b).

Bhattacharjee, C., Das, R. and Mahajan, S.M.. Phys. Rev. D 91(12), 123005 (2015).

Bodo, G.; Cattaneo, F.; Mignone, A.; Ponzo, F.; Rossi, P. Astrophys. J., 808(2), 141, (2015)

Chandrasekhar, S. Astrophys. J.74, 81 (1931); Mon. Not. R. Astron. Soc. 95, 207 (1935).

Chandrasekhar, S. An Introduction to the Study of Stellar structures, Chicago (Dover Publications, 1939).

Cheung, M. C. M., De Pontieu, B., Tarbell, T. D., Tian, Y.Fu, H., Testa, P., Reeves, K. K., Martinez-Sykora, J., Boerner, P., Wulser, J. P., Lemen, J., Title, A. M., Hurlburt, N., Kleint, L., Kankelborg, C., Jaeggli, S., Golub, L. Astrophys. J., 801, 83 (2015).

Christopoulou, E. B., Georgakilas, A. A., \& Koutchmy, S. Sol. Phys., 199, 61 (2001).

Cumming A., MNRAS, 333, 589 (2002).

Dunne, M. A high-power laser fusion facility for Europe Nature Phys. 22 (2006).

Ferrario, L., de Martino, D., \& Gaensicke, B. T. SSRv, 1 (2015).

Goodman, M. L. Astrophys. J., 533, 501 (2000); Space Sci. Rev., 95, 79 (2001).

Hansteen, V., De Pontieu, B., Carlsson, M., Lemen, J., Title, A., Boerner, P., Hurlburt, N., Tarbell, T. D. Science, 346(6207), 1255757 (2014).

Hollands, M., Gaensicke, B., \& Koester, D. MNRAS, 450, 68 (2015).

Kawka, A., Vennes, S., Schmidt, G. D., Wickramasinghe, D. T., \& Koch, R. Astrophys. J., 654, 499 (2007).

Iqbal, N., Berezhiani, V.I. and Yoshida, Z. Phys. Plasmas 15, 032905 (2008).

Kawka, A. and Vennes, S. MNRAS 439, L90 (2014).

Kepler, S. O., Pelisoli, I., Jordan, S., Kleinman, S.J., Koester, D., Külebi, D.B., Pecanha, B.V., Castanheira, B.G., Nitta, A., Costa, J.E.S., Winget, D.E., Kanaan, A. and Fraga, L. MNRAS 429, 2934 (2013). 
Koester, D. and Chanmugam,G. Rep. Prog. Phys. 53, 837 (1990).

Krishan, V. and Yoshida, Z. Phys. Plasmas, 13(9), 092303 (2006).

Külebi, B., Jordan, S., Euchner, F., Gnsicke, B. T., \& Hirsch, H. A\&A, 506, 1341 (2009).

Liebert, J., Bergeron, P., \& Holberg, J. B. AJ, 125, 348 (2003).

Mahajan, S.M. and Yoshida, Z. Phys. Rev. Lett.81, 4863 (1998).

Mahajan, S.M., Miklaszewski, R., Nikolskaya, K.I. and Shatashvili, N.L. Phys. Plasmas 8, 1340 (2001).

Mahajan, S.M., Nikolskaya, K. I., Shatashvili, N.L. and Yoshida, Z. Astrophys. J.576, L161 (2002).

Mahajan, S.M. Phys. Rev. Lett.90, 035001 (2003).

Mahajan, S.M., Shatashvili, N.L., Mikeladze, S.V. and Sigua, K.I. Astrophys. J.634, 419 (2005); Phys. Plasmas 13, 062902 (2006).

Mahajan, S.M. and Lingam, M. Phys. Plasmas, 22(9), 092123 (2015).

Mahajan, S.M. and Yoshida, Z. Phys. Rev. Lett., 105, 095005 (2010).

Michel, F.C. Theory of Neutron Star Magnetospheres, University of Chicago Press, Chicago, (1991).

Michel, F.C. Rev. Mod. Phys. 54, 1 (1982).

Morrison, P.J. Rev. Mod. Phys. 70, 467 (1998).

Mourou, G.A., Tajima, T. and Bulanov, S. V. Rev. Mod. Phys. 78309 (2006).

Ohsaki, S., Shatashvili, N.L., Yoshida, Z. and Mahajan, S.M. Astrophys. J.559, L61 (2001); Astrophys. J.570, 395 (2002).

Orlando, S., Peres, G., \& Serio, S. A\&A, 294, 861 (1995a); A\&A, 300, 549 (1995b).

Pino, J., Li, H. and Mahajan, S.M. Phys. Plasmas 17, 112112 (2010).

Sakai, J. I., \& Furusawa, K. Astrophys. J., 564, 1048 (2002).

Schrijver, C. J., et al. Sol. Phys., 187, 261 (1999).

Schmidt, G. D., Harris, H. C., Liebert, J., et al. Astrophys. J., 595, 1101 (2003).

Seaton, D.B., Winebarger, A.R., DeLuca, E.E., Golub, L., \& Reeves, K.K. Astrophys. J., 563, L173 (2001).

Shapiro, L. and Teukolsky, S.A. Black Holes, White Dwarfs and Neutron Stars: The Physics of Compact Objects, (John Wiley and Sons, New York, 1973).

Shatashvili, N.L., Mahajan, S.M. and Berezhiani, V.I. Astrophys. Space. Sci, 361, 70 (2016).

Shatashvili, N.L. and Yoshida, Z. AIP Conf. Proc. 1392, 73 (2011)

Shiraishi, J, Yoshida, Z. and Furukawa, M. Astrophys. J., 697, 100 (2009).

Shukla, P.K. and Eliasson, B. Phys. Usp. 53, 51 (2010).

Shukla, P.K. and Eliasson, B. Rev. Mod. Phys. 83, 885 (2011).

Skogsrud, H., Rouppe van der Voort, L., De Pontieu, B. and Pereira, T. M. D. Astrophys. J., 806, 170 (2015).

Socas-Navarro, H., \& Sanchez Almeida, J. Astrophys. J., 565, 1323 (2002).

Tajima, T. Eur. Phys. J. Sp. Top. 223(6), 1037 (2014).

Tian, H., DeLuca, E.E., Cranmer, S.R., De Pontieu, B., Peter, H., Martinez-Sykora, J., Golub, L. et al. Science, 346(6207), 1255711 (2014).
Tevzadze A.G., Chagelishvili G.D, Bodo G. \& Rossi P. MNRAS, 401(2), 901 (2010).

Tremblay, P.-E., Fontaine, G., Freytag, B., Steiner, O., Ludwig, H.-G., Steffen, M., Wedemeyer,S. and Brassard, P. Astrophys. J., 812, 19, (2015a).

Tremblay, P.-E., Ludwig, H.-G., Freytag, B., Fontaine, G., Steffen, M. and Brassard, P. Astrophys. J., 799, 142 (2015b).

Uchida, Y., Miyagoshi, T., Yabiku, T., Cable, S., \& Hirose, S. PASJ, 53, 331 (2001).

Winebarger, A. M., DeLuca, E. E., \& Golub, L. Astrophys. J., 553, L81 (2001). Winebarger, A. R., Warren, H., van Ballagooijen, A., DeLuca, E. E., \& Golub, L. Astrophys. J., 567, L89 (2002).

Winget, D. E. and Kepler, S. O. Annu. Rev. A\&A 46, 157 (2008).

Yanovsky, V., Chvykov, V., Kalinchenko, G., Rousseau, P., Planchon, T., Matsuoka, T., Maksimchuk, A., Nees, J., Cheriaux, G. , Mourou, G. and Krushelnick, K. Opt. Exp. 162109 (2008).

Yoshida, Z. and Mahajan, S.M. J. Math. Phys. 40, 5080 (1999).

Yoshida, Z., Mahajan, S.M., Ohsaki, S., Iqbal, M. \& Shatashvili, N.L. Phys. Plasmas, 8(5), 2125 (2001).

Zanni, C., Ferrari, A., Rosner, R., Bodo, G. and Massaglia, S. A\&A 469, 811 (2007).

This 2-column preprint was prepared with the AAS LATEX macros v5.2. 